XXXI International Workshop on High Energy Physics:

Critical Points in the Modern Particle Physics

International Journal of Modern Physics: Conference Series

Vol. 47 (2018) 1860093 (9 pages)

(C) The Author(s)

DOI: $10.1142 / \mathrm{S} 2010194518600935$

\title{
Duality and Charged Pion Condensation in Chirally Asymmetric Dense Quark Matter in the Framework of an $\mathrm{NJL}_{2}$ Model
}

\author{
T. G. Khunjua and V. C. Zhukovsky \\ Faculty of Physics, Moscow State University, 119991, Moscow, Russia \\ K. G. Klimenko and R. N. Zhokhov* \\ State Research Center of Russian Federation - Institute for High Energy Physics, NRC \\ "Kurchatov Institute", 142281, Protvino, Moscow Region, Russia \\ *zhokhovr@gmail.com
}

Published 6 June 2018

\begin{abstract}
In this talk we present investigation of the phase structure of a (1+1)-dimensional quark model with four-quark interaction and in the presence of baryon $\left(\mu_{B}\right)$, isospin $\left(\mu_{I}\right)$ and chiral isospin $\left(\mu_{I 5}\right)$ chemical potentials. Spatially homogeneous and inhomogeneous (chiral density wave (for chiral condensate) and single wave (for charged pion condensate)) condensates are considered. It is established that in the large- $N_{c}$ limit $\left(N_{c}\right.$ is the number of colored quarks) there exists a duality correspondence between the chiral symmetry breaking phase and the charged pion condensation (PC) one. The primary conclusion of this investigation is the fact that chiral isospin chemical potential generates charged pion condensation with non-zero baryon density in dense quark matter. Moreover, it is shown that inhomogeneous charged PC phase with nonzero baryon density is induced in the model by arbitrary small values of the chemical potential $\mu_{I 5}$ (for a rather large region of $\mu_{B}$ and $\mu_{I}$ ).
\end{abstract}

\section{Introduction}

QCD at nonzero temperature and baryon chemical potential plays a fundamental role in the description of a number of various physical systems. Two important ones are neutron stars, which probe the low temperature and intermediate baryon chemical potential domain, and heavy ion collision experiments, which explore the region of the high temperature and low baryon chemical potential. However, the consideration of these systems is not possible in the framework of perturbative weak coupling QCD. Calculations with nonzero baryonic chemical potential $\mu_{B}$ is very hard to be performed on the lattice as well.

This is an Open Access article published by World Scientific Publishing Company. It is distributed under the terms of the Creative Commons Attribution 4.0 (CC-BY) License. Further distribution of this work is permitted, provided the original work is properly cited. 


\section{T. G. Khunjua et al.}

To describe physical situations, when the baryonic density is nonzero usually different effective theories are employed. Among them, we especially would like to mention the NJL-type models. ${ }^{1}$ They are nonrenormalizable in $(3+1)$-dimensional spacetime and can be considered only as effective field theories.But there exist also low-dimensional theories, such as (1+1)-dimensional chiral Gross-Neveu (GN) type models, ${ }^{7,8}$ that possess a lot of common features with QCD (renormalizability, asymptotic freedom, dimensional transmutation, the spontaneous breaking of chiral symmetry) and can be used as a laboratory for the qualitative simulation of specific properties of QCD at arbitrary energies. It is well understood (see Refs. 9-11) that the usual no-go theorem, ${ }^{12}$ which generally forbids the spontaneous breaking of any continuous symmetry in two-dimensional spacetime, does not work in the limit $N_{c} \rightarrow \infty$, where $N_{c}$ is the number of colored quarks.

Besides temperature and baryon density, there are additional parameters, for instance, an isotopic chemical potential $\mu_{I}$. It allows to consider systems with isospin imbalance (different numbers of $u$ and $d$ quarks). It is realized, e.g., in neutron stars, heavy-ion experiments, etc. So QCD phase diagram in the presence of both baryonic and isotopic chemical potentials has been recently studied in Refs. 2-4, where the possibility of the charged PC phase just at $\mu_{I} \neq 0$ was predicted. However, the existence of the charged PC phase is established there without sufficient certainty. Due to these circumstances, the question arises, whether there exist any factors promoting the appearance of charged PC phenomenon in dense baryonic matter.

It was shown here in the framework of toy $\mathrm{NJL}_{2}$ model that this phase can be realized if we take into account a nonzero chiral isotopic potential in addition. This means that there should be chiral imbalance (a nonzero difference between densities of left- and right-handed fermions) in the system. Recall that chiral imbalance may arise from the chiral anomaly in the quark-gluon-plasma phase of QCD and possibly leads to the chiral magnetic effect ${ }^{16}$ in heavy-ion collisions. It might be realized also in compact stars or condensed matter systems ${ }^{17}$ (-see also the review ${ }^{18}$ ).

The existence of spatially inhomogeneous phases in dense systems is certainly not a new idea. It is very challenging to find inhomogeneous condensate as a solution and find its form analytically. However, more often one just assume some ansatz and then solve a minimax problem with respect to its parameters.

In this paper we investigate the possibility of formation of homogneous and inhomogeneous condensates in the system and charged PC phenomenon in the framework of an extended (1+1)-dimensional NJL model with two quark flavors and in the presence of the baryon $\left(\mu_{B}\right)$, isospin $\left(\mu_{I}\right)$ as well as chiral isospin $\left(\mu_{I 5}\right)$ chemical potentials. We will show that a chiral imbalance of dense and isotopically asymmetric baryon matter is a factor, which can induce there a charged PC phase.

Moreover, it has been shown in the framework of the $\mathrm{NJL}_{2}$ model under consideration that in the leading order of the large- $N_{c}$ approximation there arises a duality between chiral symmetry breaking (CSB) and charged PC phenomena. 


\section{The model and its thermodynamic potential}

We consider a two-dimensional model which is intended for simulation of the properties of real dense quark matter with two massless quark flavors ( $u$ and $d$ quarks). Its Lagrangian has the form

$$
L=\bar{q}\left[\gamma^{\nu} \mathrm{i} \partial_{\nu}+\frac{\mu_{B}}{3} \gamma^{0}+\frac{\mu_{I}}{2} \tau_{3} \gamma^{0}+\frac{\mu_{I 5}}{2} \tau_{3} \gamma^{0} \gamma^{5}\right] q+\frac{G}{N_{c}}\left[(\bar{q} q)^{2}+\left(\bar{q} \mathrm{i} \gamma^{5} \vec{\tau} q\right)^{2}\right]
$$

where the quark field $q(x) \equiv q_{i \alpha}(x)$ is a flavor doublet $(i=1,2$ or $i=u, d)$ and color $N_{c}$-plet $\left(\alpha=1, \ldots, N_{c}\right)$ as well as a two-component Dirac spinor (the summation in (1) over flavor, color, and spinor indices is implied); $\tau_{k}(k=1,2,3)$ are Pauli matrices. The quantities $\gamma^{\nu}(\nu=0,1)$ and $\gamma^{5}$ in Eq. (1) are gamma matrices in $(2+1)$-dimensional space-time. Baryon $\mu_{B}$, isospin $\mu_{I}$ and axial isospin $\mu_{I 5}$ chemical potentials are introduced in order to describe quark matter with nonzero baryon $n_{B}$, isospin $n_{I}$ and axial isospin $n_{I 5}$ densities, respectively.

To find the thermodynamic potential of the system, we use a semi-bosonized version of the Lagrangian (1)

$$
\widetilde{L}=\bar{q}\left[\gamma^{\rho} \mathrm{i} \partial_{\rho}+\mu \gamma^{0}+\nu \tau_{3} \gamma^{0}+\nu_{5} \tau_{3} \gamma^{1}-\sigma-\mathrm{i} \gamma^{5} \pi_{a} \tau_{a}\right] q-\frac{N_{c}}{4 G}\left[\sigma \sigma+\pi_{a} \pi_{a}\right] .
$$

Starting from the theory (2), one obtains in the leading order of the large $N_{c^{-}}$ expansion (i.e. in the one-fermion loop approximation) the following path integral expression for the effective action $\mathcal{S}_{\text {eff }}\left(\sigma, \pi_{a}\right)$ of the bosonic $\sigma(x)$ and $\pi_{a}(x)$ fields:

$$
\exp \left(\mathrm{i} \mathcal{S}_{\text {eff }}\left(\sigma, \pi_{a}\right)\right)=N^{\prime} \int[d \bar{q}][d q] \exp \left(\mathrm{i} \int \widetilde{L} d^{2} x\right)
$$

$N^{\prime}$ is a normalization constant. The ground state expectation values $\langle\sigma(x)\rangle$ and $\left\langle\pi_{a}(x)\right\rangle$ of the composite bosonic fields are determined by the saddle point equations,

$$
\frac{\delta \mathcal{S}_{\text {eff }}}{\delta \sigma(x)}=0, \quad \frac{\delta \mathcal{S}_{\text {eff }}}{\delta \pi_{a}(x)}=0,
$$

We use the following spatially inhomogeneous CDW ansatz for chiral condensate and the single plane wave ansatz for charged pion condensates:

$$
\begin{aligned}
& \langle\sigma(x)\rangle=M \cos (2 k x), \quad\left\langle\pi_{3}(x)\right\rangle=M \sin (2 k x), \\
& \left\langle\pi_{1}(x)\right\rangle=\Delta \cos \left(2 k^{\prime} x\right), \quad\left\langle\pi_{2}(x)\right\rangle=\Delta \sin \left(2 k^{\prime} x\right),
\end{aligned}
$$

where gaps $M, \Delta$ and wavevectors $k, k^{\prime}$ are constant dynamical quantities.

In the leading order of the large $N_{c}$-expansion after performing the so-called Weinberg (or chiral) transformation it is possible to find for the TDP the following expression:

$$
\Omega\left(M, k, k^{\prime}, \Delta\right)=\frac{M^{2}+\Delta^{2}}{4 G}+\mathrm{i} \int \frac{d^{2} p}{(2 \pi)^{2}} \ln P_{4}\left(p_{0}\right)
$$


T. G. Khunjua et al.

In Eq. (5) we use the notations $P_{4}\left(p_{0}\right)=\epsilon_{1} \epsilon_{2} \epsilon_{3} \epsilon_{4}=\eta^{4}-2 a \eta^{2}-b \eta+c$, where $\eta=p_{0}+\mu$ and

$$
\begin{aligned}
& a=M^{2}+\Delta^{2}+p_{1}^{2}+\tilde{\nu}^{2}+\tilde{\nu}_{5}^{2} ; \quad b=8 p_{1} \tilde{\nu} \tilde{\nu}_{5} ; \\
& c=a^{2}-4 p_{1}^{2}\left(\tilde{\nu}^{2}+\tilde{\nu}_{5}^{2}\right)-4 M^{2} \tilde{\nu}^{2}-4 \Delta^{2} \tilde{\nu}_{5}^{2}-4 \tilde{\nu}^{2} \tilde{\nu}_{5}^{2} .
\end{aligned}
$$

It is evident that the TDP is an even function over each of the variables $M$ and $\Delta$ as well as over $\mu, \tilde{\nu}, \tilde{\nu}_{5}$. Hence,we can consider in the following only $\mu \geq 0, \tilde{\nu} \geq 0$, $\tilde{\nu}_{5} \geq 0, M \geq 0$, and $\Delta \geq 0$ values of these quantities. Moreover, the expression (5) for the TDP is invariant with respect to the so-called duality transformation,

$$
\mathcal{D}: \quad M \longleftrightarrow \Delta, \quad \nu \longleftrightarrow \nu_{5}, \quad k \longleftrightarrow k^{\prime}
$$

It means that in the leading order of the large- $N_{c}$ approximation there is the socalled duality correspondence between chiral symmetry breaking (CSB) and charged PC phenomena.

\subsection{Thermodynamic potential}

It can be shown numerically that GMP of the TDP can never be of the form $\left(M_{0} \neq\right.$ $\left.0, \Delta_{0} \neq 0\right)$. Hence, in order to establish the phase portrait of the model, it is enough to study the projections $F_{1}(M) \equiv \Omega^{r e n}(M, \Delta=0)$ and $F_{2}(\Delta) \equiv \Omega^{r e n}(M=0, \Delta)$ of the TDP to the $M$ and $\Delta$ axes, correspondingly. It is possible to obtain the following expressions for these quantities in the case $k=0, k^{\prime}=0$,

$$
\begin{aligned}
& F_{1}(M)=\frac{M^{2}}{2 \pi} \ln \left(\frac{M^{2}}{m^{2}}\right)-\frac{M^{2}}{2 \pi}-\frac{\nu_{5}^{2}}{\pi}-\theta(\mu+\nu-M) \frac{\mathcal{A}}{\pi} \\
& -\theta(|\mu-\nu|-M) \theta\left(\sqrt{(\mu-\nu)^{2}-M^{2}}-\nu_{5}\right) \frac{\mathcal{B}}{2 \pi} \\
& +\theta(\mu+\nu-M) \theta\left(\sqrt{(\mu+\nu)^{2}-M^{2}}-\nu_{5}\right) \frac{\mathcal{C}}{2 \pi},
\end{aligned}
$$

where

$$
\begin{gathered}
\mathcal{A}=(\mu+\nu) \sqrt{(\mu+\nu)^{2}-M^{2}}-M^{2} \ln \frac{\mu+\nu+\sqrt{(\mu+\nu)^{2}-M^{2}}}{M} \\
\mathcal{B}=|\mu-\nu| \sqrt{(\mu-\nu)^{2}-M^{2}}+\nu_{5} \sqrt{\nu_{5}^{2}+M^{2}} \\
-2|\mu-\nu| \nu_{5}-M^{2} \ln \frac{|\mu-\nu|+\sqrt{|\mu-\nu|^{2}-M^{2}}}{\nu_{5}+\sqrt{\nu_{5}^{2}+M^{2}}} \\
\mathcal{C}=(\mu+\nu) \sqrt{(\mu+\nu)^{2}-M^{2}}+\nu_{5} \sqrt{\nu_{5}^{2}+M^{2}}-2(\mu+\nu) \nu_{5} \\
-M^{2} \ln \frac{\mu+\nu+\sqrt{(\mu+\nu)^{2}-M^{2}}}{\nu_{5}+\sqrt{\nu_{5}^{2}+M^{2}}}
\end{gathered}
$$




$$
F_{2}(\Delta)=\left.F_{1}(\Delta)\right|_{\nu \longleftrightarrow \nu_{5}} .
$$

The TDP with $k, k^{\prime} \neq 0 \Omega^{u n}\left(M, k, k^{\prime}, \Delta\right)$ can be obtained from the TDP in the homogeneous case simply performing there the replacement $\nu, \nu_{5} \rightarrow \tilde{\nu} \equiv \nu+k, \tilde{\nu_{5}} \equiv$ $\nu_{5}+k^{\prime}$. However, the TDP has several unphysical properties such as (i) the unboundedness from below with respect to the variables $k, k^{\prime}$. (The unboundedness from below of the TDP is evident, e.g., from the expression (8) if $\nu_{5} \rightarrow \tilde{\nu}_{5}$. (ii) Moreover, one can observe immediately that at $M=0$ and $\Delta=0$ the expression for the thermodynamic potential does depend on $k$ and $k^{\prime}$. Bearing in mind the expression (4) it is obvious that this is also quite unphysical and we need to change somehow the expression for thermodynamic potential in such a way that this dependence is eliminated.

The above mentioned nonphysical properties should be eliminated by, e.g., the subtraction operation applying twice, first with respect to the variables $M, k$ and then with respect to $\Delta, k^{\prime}$. As a result, we have the following physically relevant TDP,

$$
\begin{aligned}
& \Omega^{\text {phys }}\left(M, k, k^{\prime}, \Delta\right)=\Omega^{r e n}\left(M, k, k^{\prime}, \Delta\right)-\Omega^{r e n}\left(M, k, k^{\prime}, 0\right)+\Omega^{r e n}(M, k, 0,0) \\
& -\Omega^{r e n}\left(0, k, k^{\prime}, \Delta\right)+\Omega^{r e n}\left(0,0, k^{\prime}, \Delta\right)-\Omega^{r e n}(0, k, 0,0) \\
& -\Omega^{r e n}\left(0,0, k^{\prime}, 0\right)+\Omega^{r e n}\left(0, k, k^{\prime}, 0\right)+\Omega^{r e n}(0,0,0,0) .
\end{aligned}
$$

It turns out that the mixed phase $\left(\left(M_{0} \neq 0, \Delta_{0} \neq 0\right)\right)$ is absent in the model in inhomogeneous case as well, so it is enough to study only the projections of the TDP on the $M$ and $\Delta$ axes.

There could be several phases in the model (1). The first one is the symmetric phase with zero gaps $M_{0}=0, \Delta_{0}=0$ and zero values of the wavevectors $k_{0}=$ $0, k_{0}^{\prime}=0$. In the chiral symmetry breaking CSB (in the charged pion condensation CPC) phase the TDP reaches the least value at the global minimum point with $M_{0} \neq 0, \Delta_{0}=0$ or $\left(M_{0}=0, \Delta_{0} \neq 0\right.$.

\subsection{Phase diagrams and duality property of the model}

In Fig. 1 the $\left(\mu, \nu, \nu_{5}\right)$-phase portrait of the model is presented in the supposition that all condensates are spatially homogeneous. ${ }^{15}$ It is clear from this figure that charged PC phase with nonzero quark number density $n_{q}$ (this phase is denoted there by PCd) can be realized in the model (1) only at rather large values of $\nu_{5}$. Now let us discuss the the case of spatially inhomogeneous condensates

\subsubsection{Different $(\mu, \nu)$-phase diagrams}

At infinitesimal values (see Fig. 2) of $\nu_{5}$ if one looks at the region of $\mu>m / \sqrt{2}$ one can see there ICSBd phase (symbol " $\mathrm{d}$ " means that quark number density is nonzero), at lower values of $\mu$ there is a region which is called "Mixed inhomogeneous 


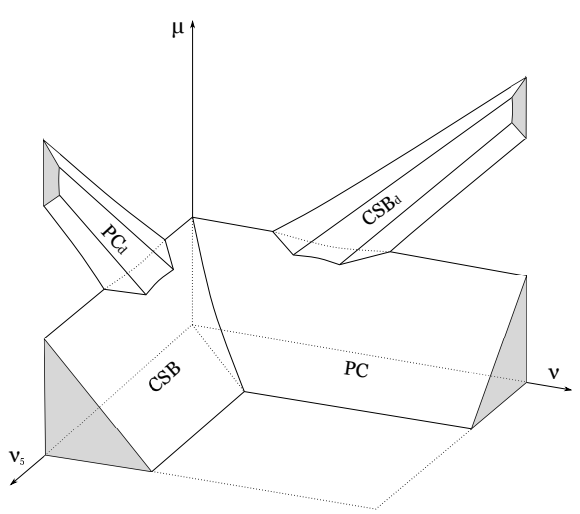

Fig. 1. Schematic representation of the $\left(\nu_{5}, \nu, \mu\right)$-phase portrait of the model in the case of spatially homogeneous condensates.

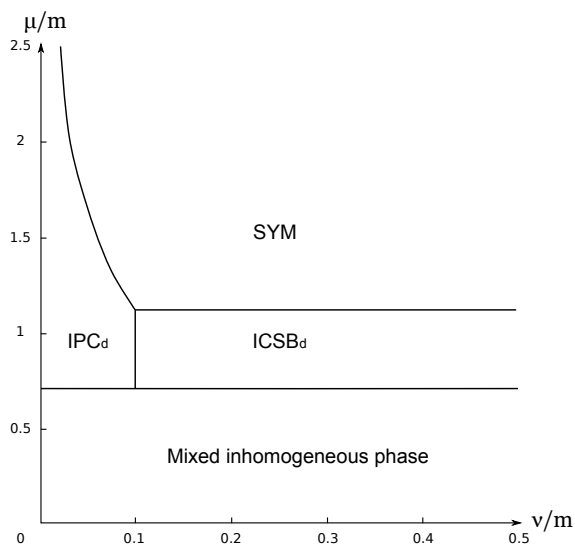

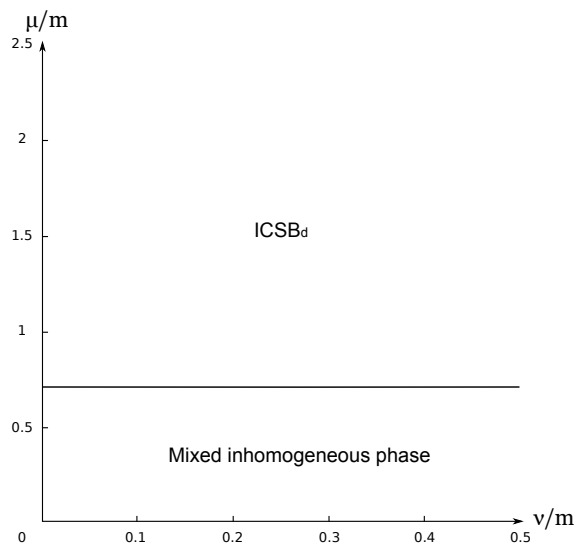

Fig. 2. The case of inhomogeneous condensates: The $(\nu, \mu)$-phase portrait at $\nu_{5}=0_{+}$.

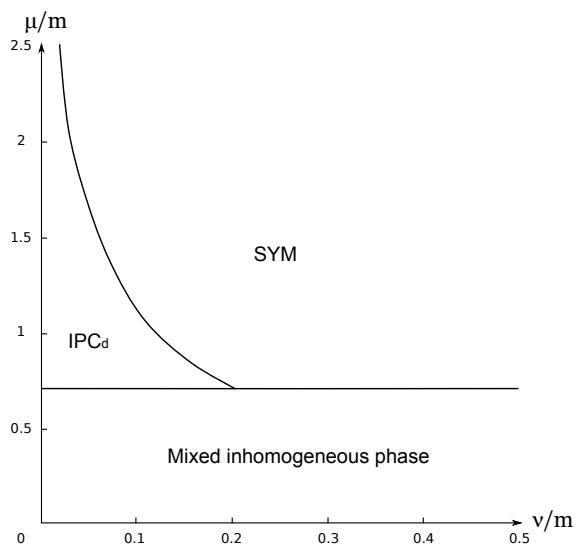

Fig. 3. The case of spatially inhomogeneous condensates: The $(\nu, \mu)$-phase portrait of the model at $\nu_{5}=0.1 \mathrm{~m}$ (left figure) and at $\nu_{5} \geq 0.2 \mathrm{~m}$ (right figure).

phase". It turns out that for each point $(\mu, \nu)$ belonging to this region the TDP has two degenerate global minima, first of them corresponds to ICSB phase, the second to inhomogeneous charged pion condensation (IPC) phase.

The structure of $(\mu, \nu)$-phase diagrams at other fixed values of the chiral chemical potential $\nu_{5}$ can be easily understood from the phase portraits of Figs. 3, where $(\mu, \nu)$-phase diagrams are presented for two qualitatively different values of $\nu_{5}$. It is clear from the figure that at each finite $\nu_{5}>0$ the $(\mu, \nu)$-phase diagram contains IPCd phase. Moreover, the greater $\nu_{5}$, the smaller the size of the ICSBd phase, which disappears from a $(\mu, \nu)$-phase portrait at $\nu_{5} \geq 0.2 \mathrm{~m}$. Hence, in the framework of 


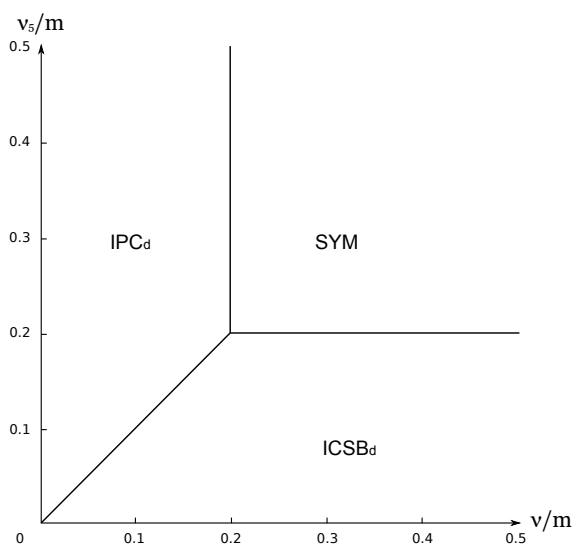

Fig. 4. The case of spatially inhomogeneous condensates: The $\left(\nu, \nu_{5}\right)$-phase portrait of the model at $\mu=0.75 \mathrm{~m}$. All notations are described in Figs. 2, 3.

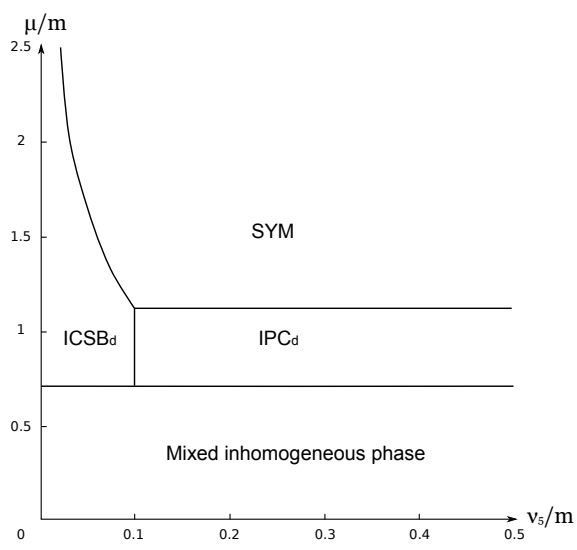

Fig. 5. The case of spatially inhomogeneous condensates: The $\left(\nu_{5}, \mu\right)$-phase portrait of the model at $\nu=0.1 \mathrm{~m}$. All notations are described in Figs. 2, 3.

the initial $\mathrm{NJL}_{2}$ model, the chiral chemical potential $\nu_{5}$ serves as a factor, which promotes the charged pion condensation phenomenon in dense quark matter (it is the IPCd phase in all figures).

\subsubsection{Other phase diagrams and the role of duality}

Let us discuss the role of the duality (7) of the TDP on the phase structure. Suppose that at some fixed $\mu, \nu=A$ and $\nu_{5}=B$ point CSB phase is realized in the model. Then it follows from the duality of the TDP that at permuted chemical potential values (i.e. at $\nu=B$ and $\nu_{5}=A$ and the same $\mu$ ) PC phase is realized (and vice versa). This is the so-called duality correspondence between CSB and charged PC phases.

Under the duality transformation the most general $\left(\nu, \nu_{5}, \mu\right)$-phase portrait is mapped to itself (self-dual). Likewise at arbitrary fixed $\mu$ the $\left(\nu, \nu_{5}\right)$-phase diagram of the model is also self-dual. These conclusions are supported by Fig. 4. If $\mu<$ $m / \sqrt{2}$, then the $\left(\nu, \nu_{5}\right)$-phase portrait is even simpler because at each point of it the "Mixed inhomogeneous phase" is realized.

Now let us show how to construct the $\left(\nu_{5}, \mu\right)$-phase diagram of the model at arbitrary fixed value $\nu=A$ using duality transformation. For example, to find the $\left(\nu_{5}, \mu\right)$-phase diagram at $\nu=0.1 \mathrm{~m}$ we should start from the $(\nu, \mu)$-diagram at fixed $\nu_{5}=0.1 \mathrm{~m}$ of Fig. 3 (left panel) and make the replacement $: \nu \leftrightarrow \nu_{5}, \mathrm{IPCd} \leftrightarrow \mathrm{ICSBd}$. As a result we obtain the phase diagram of Fig. 5 . In a similar way one can dually transform Fig. 2 and Fig. 3 (right panel) in order to find the $\left(\nu_{5}, \mu\right)$-phase diagrams at $\nu=0_{+}$and $\nu \geq 0.2 \mathrm{~m}$, respectively, etc. 


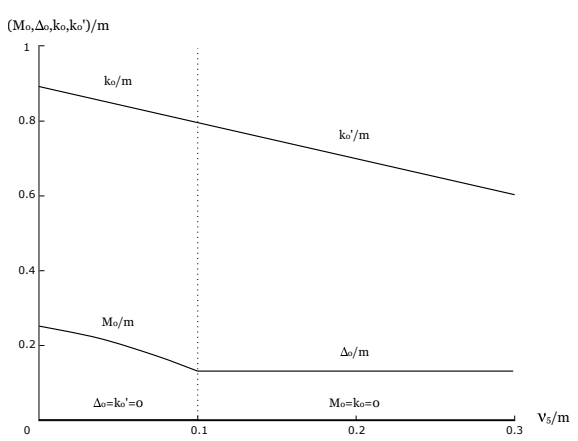

Fig. 6. The case of spatially inhomogeneous condensates: The behavior of the coordinates $M_{0}, k_{0}, k_{0}^{\prime}, \Delta_{0}$ of the GMP of the TDP (13) as functions of $\nu_{5}$ for fixed $\mu=m$ and $\nu=0.1 m$.

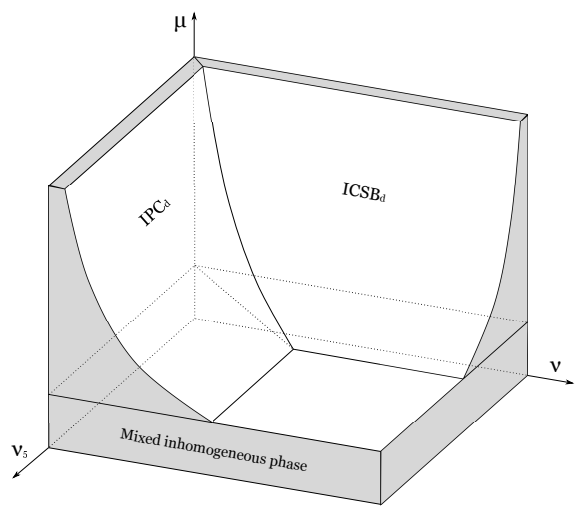

Fig. 7. Schematic representation of the $\left(\nu_{5}, \nu, \mu\right)$-phase portrait of the model in the case of spatially inhomogeneous condensates.

The behaviour of different order parameters are presented in Fig. 6. It is clear from the figure that in the critical point $\nu_{5}=0.1 \mathrm{~m}$ there is a phase transition in the system from ICSBd phase to IPCd phase.

The most general phase portrait $\left(\nu, \nu_{5}, \mu\right)$ is presented schematically at Fig. 7. As is easily seen from this figure the phase diagram is self-dual. Moreover, it supports the above conclusion: the charged PC phenomenon can be realized in chirally asymmetric quark matter with nonzero baryon density.

\section{Summary and Conclusions}

In this talk the phase structure of the $\mathrm{NJL}_{2}$ model (1) with two quark flavors is investigated in the large- $N_{c}$ limit in the presence of baryon $\mu_{B}$, isospin $\mu_{I}$ and chiral isospin $\mu_{I 5}$ chemical potentials. Let us summarize some of the most interesting results obtained.

1) The chemical potential $\mu_{I 5}$ generates charged pion condensation in dense quark matter.

2) In this model inhomogeneous condensates are quite favoured compared to homogeneous condensates.

3) We demonstrated in the framework of the $\mathrm{NJL}_{2}$ model (1), that in the leading order of the large- $N_{c}$ approximation there is duality correspondence between CSB and charged PC phenomena.

\section{References}

1. Y. Nambu and G. Jona-Lasinio, Phys. Rev. D 112, 345 (1961).

2. D.T. Son and M.A. Stephanov, Phys. Atom. Nucl. 64, 834 (2001); M. Loewe and C. Villavicencio, Phys. Rev. D 67, 074034 (2003); M. Loewe and C. Villavicencio, "Pion 
stability in a hot dense media", arXiv:1107.3859; L. He, M. Jin, and P. Zhuang, Phys. Rev. D 71, 116001 (2005); D.C. Duarte, R.L.S. Farias and R.O. Ramos, Phys. Rev. D 84, 083525 (2011); D. Ebert, K.G. Klimenko, A.V. Tyukov and V.C. .Zhukovsky, Eur. Phys. J. C 58, 57 (2008).

3. D. Ebert and K.G. Klimenko, J. Phys. G 32, 599 (2006); Eur. Phys. J. C 46, 771 (2006).

4. J.O. Andersen and T. Brauner, Phys. Rev. D 78, 014030 (2008); J.O. Andersen and L. Kyllingstad, J. Phys. G 37, 015003 (2009); Y. Jiang, K. Ren, T. Xia and P. Zhuang, "Meson Screening Mass in a Strongly Coupled Pion Superfluid," arXiv:1104.0094.

5. C.f. Mu, L.y. He and Y.x. Liu, Phys. Rev. D 82, 056006 (2010).

6. J.O. Andersen, W.R. Naylor and A. Tranberg, Rev. Mod. Phys. 88, 025001 (2016).

7. D.J. Gross and A. Neveu, Phys. Rev. D 10, 3235 (1974).

8. J. Feinberg, Annals Phys. 309, 166 (2004); M. Thies, J. Phys. A 39, 12707 (2006).

9. A. Barducci, R. Casalbuoni, R. Gatto, M. Modugno, and G. Pettini, Phys. Rev. D 51, 3042 (1995).

10. A. Chodos, H. Minakata, F. Cooper, A. Singh, and W. Mao, Phys. Rev. D 61, 045011 (2000); K. Ohwa, Phys. Rev. D 65, 085040 (2002).

11. V. Schon and M. Thies, Phys. Rev. D 62, 096002 (2000); A. Brzoska and M. Thies, Phys. Rev. D 65, 125001 (2002).

12. N.D. Mermin and H. Wagner, Phys. Rev. Lett. 17, 1133 (1966); S. Coleman, Commun. Math. Phys. 31, 259 (1973).

13. D. Ebert, N.V. Gubina, K.G. Klimenko, S.G. Kurbanov, V.C. Zhukovsky, Phys. Rev. D 84, 025004 (2011).

14. D. Ebert and K.G. Klimenko, "Pion condensation in the Gross-Neveu model with nonzero baryon and isospin chemical potentials," arXiv:0902.1861 [hep-ph].

15. D. Ebert, T.G. Khunjua and K.G. Klimenko, Phys. Rev. D 94, 116016 (2016) [arXiv:1608.07688].

16. K. Fukushima, D.E. Kharzeev and H.J. Warringa, Phys.Rev.D 78, 074033 (2008).

17. A.A. Andrianov, D. Espriu and X. Planells, Eur. Phys. J. C 73, 2294 (2013); Eur. Phys. J. C 74, 2776 (2014); R. Gatto and M. Ruggieri, Phys. Rev. D 85, 054013 (2012); L. Yu, H. Liu and M. Huang, Phys. Rev. D 90, 074009 (2014); L. Yu, H. Liu and M. Huang, Phys. Rev. D 94, 014026 (2016); G. Cao and P. Zhuang, Phys. Rev. D 92, 105030 (2015); V.V. Braguta and A.Y. Kotov, Phys. Rev. D 93, no. 10, 105025 (2016); M. Ruggieri and G. X. Peng, arXiv:1602.05250 [hep-ph].

18. V.A. Miransky and I.A. Shovkovy, Phys. Rept. 576, 1 (2015).

19. G. Gruner, Rev. Mod. Phys. 66, 1 (1994).

20. P. Fulde, R.A. Ferrell, Phys. Rev. A 135, 550 (1964).

21. A.I. Larkin, Y.N. Ovchinnikov, Zh. Eksp. Teor. Fiz. 47, 1136 (1964) [Sov. Phys. JETP 20, $762(1965)]$.

22. S. Weinberg, "The quantum Theory of Field II", Cambridge Univ. Press, Cambridge, England, 1996.

23. K. Fujikawa, Phys. Rev. D 21, 2848 (1980).

24. C. Vafa and E. Witten, Nucl. Phys. B 234, 173 (1984).

25. E.V. Gorbar, M. Hashimoto and V.A. Miransky, Phys. Rev. Lett. 96, 022005 (2006); J.O. Andersen and T. Brauner, Phys. Rev. D 81, 096004 (2010); C.f. Mu, L.y. He and Y.x. Liu, Phys. Rev. D 82, 056006 (2010).

26. G. Birkhoff and S. Mac Lane, "A Survey of Modern Algebra", New York: Macmillan, 1977. 